\title{
Development of a rat model to assess the efficacy of the somatosensory-evoked potential as indicator of analgesia
}

\author{
H. van Oostrom ${ }^{\mathrm{a}, *}$, P.J. Stienen ${ }^{\mathrm{a}}$, R. van den Bos ${ }^{\mathrm{c}}$, H.N.M. de Groot ${ }^{\mathrm{a}}$, L.J. Hellebrekers ${ }^{\mathrm{a}, \mathrm{b}}$ \\ ${ }^{a}$ Department of Clinical Sciences of Companion Animals, Section Anaesthesiology, Faculty of Veterinary Medicine, Utrecht University, \\ P.O. Box 80.154 Yalelaan 8, NL-3508 TD Utrecht, The Netherlands \\ ${ }^{\mathrm{b}}$ Department of Equine Sciences, Section Anaesthesiology, Faculty of Veterinary Medicine, Utrecht University, \\ P.O. Box 80.153 Yalelaan 12, NL-3508 TD Utrecht, The Netherlands \\ ${ }^{\mathrm{c}}$ Department of Animals, Science and Society, Section Ethology and Welfare, Faculty of Veterinary Medicine, Utrecht University, \\ P.O. Box 80.166 Yalelaan 2, NL-3508 TD Utrecht, The Netherlands
}

Accepted 8 February 2005

Available online 10 March 2005

\begin{abstract}
Drug-induced changes in somatosensory-evoked potentials (SEPs) are considered to reflect an altered nociceptive state. Therefore, the SEP is proposed to be a parameter of analgesic efficacy. However, at present, SEPs have not been studied in relation to animal pain. The present study aims to develop a rat model in which this relationship can be studied based on Pavlovian fear conditioning. Therefore, rats, implanted with epidural electro-encephalogram recording electrodes, were randomly assigned to either a paired or random-control group and subjected to an aversive-to-appetitive transfer paradigm. During the aversive phase, the SEP-stimulation paradigm (5 mA square wave pulses, $n=72$, of $2 \mathrm{~ms}$ duration each, with a stimulus frequency of $0.5 \mathrm{~Hz}$; total duration $144 \mathrm{~s}$ ) was used as the unconditioned stimulus (US), while a tone $(40 \mathrm{~s}, 1500 \mathrm{~Hz}, 85 \mathrm{~dB}$ sound pressure level) was used as the conditioned stimulus (CS). During the appetitive phase, the CS was presented paired to the presentation of a sugar pellet. When compared to the random-control group, the paired group showed significantly more freezing behavior and significantly less reward-directed behavior in response to the CS in the appetitive phase. In addition, SEPs were not significantly affected by fear conditioning. Based on these results, we conclude that the SEP-stimulation paradigm can be successfully employed as a US in fear conditioning. In future studies, fear conditioning can be carried out under different levels of an analgesic regimen to allow the changes in SEP parameters to be compared to changes in fear-induced behavior making this model potentially useful to validate SEP parameters as indicators of analgesia.
\end{abstract}

(C) 2005 Elsevier B.V. All rights reserved.

Theme: Neural basis of behavior

Topic: Learning and memory: systems and functions—animals

Keywords: SEP; Fear conditioning; Aversive-to-appetitive transfer paradigm; Somatosensory system; Analgesic efficacy; Nociception

\section{Introduction}

The somatosensory-evoked potential (SEP) is a fragment of electroencephalogram, recorded time-locked to a somatosensory stimulus. Averaging several recordings eliminates unspecific electroencephalographic signals, leaving only the stimulus-related electroencephalogram, the SEP. The SEP

\footnotetext{
* Corresponding author. Fax: +31 302518126.

E-mail address: H.vanOostrom@vet.uu.nl (H. van Oostrom).
}

evoked after high intensity stimulation of peripheral somatosensory fibers is believed to represent the neural processing of noxious stimuli.

Although in the rat, SEP components in the $10-30 \mathrm{~ms}$ latency range have been largely neglected with respect to nociception and analgesia in the literature, it has been shown that SEP components in this latency range may be of special interest in this respect. A positive peak occurring at approximately $15 \mathrm{~ms}$ (P15) recorded from the vertex (Vx-SEP) has been shown to be highly sensitive to increased stimulus frequency 
and different anesthetic drugs $[14,15]$. These findings have led to our working hypothesis that the Vx-SEP P15 reflects a primary somatosensory mechanism to discriminate between relevant and irrelevant somatosensory stimuli rather than somatosensation per se. Consequently, the Vx-SEP P15 is suggested to be of special interest in relation to nociception and analgesia. However, it remains unknown whether experimental-induced changes in the Vx-SEP waveform also involve emotional components rather than just nociception.

The International Association for the Study of Pain (IASP) defines analgesia as absence of pain in response to stimulation that would normally be painful. Therefore, when the Vx-SEP P15 is to be considered of special interest in relation to analgesia, the stimuli applied to evoke this signal (the SEP-stimulation paradigm) must be painful under normal conditions. Since the IASP definition of pain (an unpleasant sensory and emotional experience associated with actual or potential tissue damage or described in terms of such damage) involves an emotional component, the important question is whether the SEP-stimulation paradigm is indeed experienced as emotionally unpleasant. Consequently, the next important question is whether the changes in the Vx-SEP P15 are indeed related to changes in the unpleasant emotional experience of the SEP-stimulation paradigm (i.e. changes in perception of pain).

To our knowledge, no reports have become available to this date presenting data comparing changes in Vx-SEP components and changes in animal pain. This fundamental relationship needs to be defined before firm conclusions can be drawn with respect to the Vx-SEP P15 as potential indicator of analgesic efficacy. This study aimed to develop a rat model based on Pavlovian fear conditioning in which the correlation between Vx-SEP components and animal pain can be investigated.

During Pavlovian fear conditioning, animals learn that an innocuous stimulus, the conditioned stimulus (CS), predicts an aversive stimulus, the unconditioned stimulus (US). After several CS-US pairings, the CS will evoke fear-conditioned behavior. The level of fear-conditioned behavior, expressed after presenting the CS, correlates with the intensity of the shock used as the US [1]. Therefore, the amount of fearconditioned behavior is considered to be a measure for the animal's emotional experience of the aversive stimulus. When the SEP-stimulation paradigm is employed as a US in fear-conditioning sessions, the Vx-SEP can be recorded simultaneously during these sessions. When fear-conditioning different groups of rats under different levels of an analgesic regimen, the relationship between the drug-induced changes in the Vx-SEP components and animal pain can be studied by assessing the amount of fear-conditioned behavior. This approach is potentially useful to validate the Vx-SEP P15 as parameter of the level of analgesia.

Most Pavlovian fear-conditioning studies use a single aversive stimulus as US, most often a foot-shock. Contrary to this, a SEP-stimulation paradigm consists of repeated stimuli over a longer time span. Therefore, we needed to determine whether the SEP-stimulation paradigm provides an effective US in a fear-conditioning paradigm. In the present study, the effectiveness and applicability of the SEPstimulation paradigm as a US in a Pavlov fear-conditioning paradigm were investigated. Furthermore, it was determined whether fear conditioning affected the Vx-SEP components. To this goal, rats were subjected to an aversive-to-appetitive transfer paradigm.

An aversive-to-appetitive transfer consists of two phases. During Phase 1, i.e. the aversive phase, the animal is subjected to a Pavlovian fear-conditioning paradigm in which a CS is presented paired with an aversive US. During Phase 2, i.e. the appetitive phase, the same CS is presented paired with an appetitive US, most often a food or water reward. In such a transfer paradigm, the aversive association with the CS made during Phase 1 impedes the appetitive response during Phase 2. Both the aversive behavioral responses and the acquisition rate of the appetitive responses shown after CS presentations during Phase 2 can be used as parameters for the strength of the association between the CS and the aversive US formed during Phase 1 [2]. In this study, Phase 1 consisted of 10 Pavlovian fear-conditioning trials in which the SEPstimulation paradigm ( $5 \mathrm{~mA}$ square wave pulses, $n=72$, of $2 \mathrm{~ms}$ duration each, with a stimulus frequency of $0.5 \mathrm{~Hz}$; total time $144 \mathrm{~s}$ ) was presented either paired (paired group) or randomly (random-control group) to the CS (a 40 s 1500 $\mathrm{Hz}$ tone, $85 \mathrm{~dB}$ sound pressure level). SEPs were recorded for every US presentation. For both groups, Phase 2 consisted of 4 subsequent days, on which the CS was presented paired with a sugar pellet for 10 trials a day. During these 4 days, the strength of the association between the CS and the SEP-stimulation paradigm was determined by studying the aversive behavioral response (freezing behavior) as well as reward-directed behavior (latencies to, and number of, food magazine-visits).

\section{Material and methods}

\subsection{Animals and surgery}

Animal care and experimentation were performed in accordance with protocols approved by the Science Committee and the local Animal Experimentation Committee (Utrecht University, Utrecht, The Netherlands).

Adult male Wistar rats (HsdCpb:WU, Harlan Netherlands BV, Zeist, body weight 300-350 g, $n=20$ ) were anesthetized with $0.3 \mathrm{mg} / \mathrm{kg}$ fentanyl (i.p., Fentanyl Janssen ${ }^{\circledR}$, Janssen-Cilag BV, Tilburg, The Netherlands, containing $0.05 \mathrm{mg} / \mathrm{ml}$ fentanyl citrate) and $0.3 \mathrm{mg} / \mathrm{kg}$ medetomidine (i.p., Domitor ${ }^{\circledR}$, Pfizer Animal Health BV, Capelle a/d IJssel, The Netherlands, containing $1 \mathrm{mg} / \mathrm{ml}$ medetomidine hydrochloride) and fixed in a stereotaxic apparatus (Model 963, Ultra Precise Small Animal Stereotaxic, David Kopf Instruments, Tujunga, CA, USA). 
Epidural electrodes (wired stainless steel screws, tip diameter $0.6 \mathrm{~mm}$, impedance 300-350 $\Omega$, Fabory DIN 84A-A2, Borstlap BV, Tilburg, The Netherlands) were implanted at the vertex $(4.5 \mathrm{~mm}$ caudal to bregma, $1.0 \mathrm{~mm}$ right from midline) and bilateral in the frontal sinus (10.0 mm rostral to bregma, $1.0 \mathrm{~mm}$ left and right from midline, respectively) [14]. All electrodes were wired to an eight-pin receptacle (Mecap Preci-Dip 917-93-108-41-005, Preci-Dip Durtal SA, Delémont, Switzerland) and fixed to the skull with dental cement (Simplex Rapid, Associated Dental Products, Ltd, Swindon, UK). At the end of surgery, anesthesia was antagonized with $1 \mathrm{mg} / \mathrm{kg}$ atipamezole (s.c., Antisedan ${ }^{\circledR}$, Pfizer Animal Health BV, Capelle a/d IJssel, The Netherlands, containing $5 \mathrm{mg} / \mathrm{ml}$ atipamezole hydrochloride) and $0.15 \mathrm{mg} / \mathrm{kg}$ buprenorphine (s.c., Temgesic ${ }^{\circledR}$, Schering-Plough, Amstelveen, The Netherlands, containing $0.3 \mathrm{mg} / \mathrm{ml}$ buprenorphine). Postoperative analgesia was provided by $0.15 \mathrm{mg} / \mathrm{kg}$ buprenorphine, administered s.c. at $8 \mathrm{~h}$ intervals for 3 days after surgery.

After surgery, the animals were housed individually in clear plastic cages under climate-controlled conditions on an inversed 12:12 h light/dark cycle (lights on at 18:00 h), with ad libitum access to food and tap water. Animals were allowed to recover for at least 2 weeks prior to the start of the experiments.

\subsection{Aversive-to-appetitive transfer paradigm}

\subsubsection{Phase 1: apparatus}

Fear-conditioning sessions were performed in a darkened experimental room lit by a single $25-\mathrm{W}$ red light bulb. Ventilators in the room produced a constant background noise of approximately $70 \mathrm{~dB}$ sound pressure level. The fearconditioning box was made of Plexiglas $(40 \times 28 \times 30 \mathrm{~cm})$ with a grounded stainless-steel bottom, surrounded by a Faraday cage. The box was shielded by polystyrene plates preventing the animals from getting visual cues from outside the fear-conditioning box, only leaving access to a camera for observation of the animal's behavior. The CS (a 40 s 1500 $\mathrm{Hz}$ tone, $85 \mathrm{~dB}$ sound pressure level) was generated by a sound generator (33120 A, Arbitrary Waveform Generator, Hewlett Packard, Palo Alto, CA, USA) and presented by two speakers mounted in the covering lid of the box. The US (5 $\mathrm{mA}$ square wave pulses, $n=72$, of $2 \mathrm{~ms}$ duration each, with a stimulus frequency of $0.5 \mathrm{~Hz}$; total time $144 \mathrm{~s}$ ) was generated by a Grass-stimulator (Model S-88, Grass Medical Instruments, Quincy, Mass, USA) and delivered to a Grass stimulation isolation unit (Model SUI 5, Grass Medical Instruments) and constant current unit (Model CCU 1A, Grass Medical Instruments) to control the intensity.

\subsubsection{Phase 1: procedure}

The animals were randomly assigned to either the paired or the random-control group. All fear-conditioning sessions were performed in the "lights off" period. Approximately 30 min prior to the start of the fear-conditioning session, the animals were placed in the experimental room to acclimatize. Next, they were fitted in a tight fitting jacket allowing free movement, and an electrical stimulation device was fixed at the left tail base. Subsequently, the animals were placed in the fear-conditioning box. The stimulation device was wired to the Grass-stimulator via a swivel connector. The receptacle at the animal's head was wired via the same swivel-connector to the recording device (described in Section 2.3). This approach allowed free movement of the animal during the session as described by Stienen et al. [14]. The fear-conditioning session started after $15 \mathrm{~min}$ of acclimatization in the box.

The paired group was subjected to a Pavlovian fearconditioning paradigm in which the CS was presented paired with the US. The US started $10 \mathrm{~s}$ after the CS, creating a $30 \mathrm{~s}$ overlap between CS and US. The interval between the CS onsets was $454 \mathrm{~s}$. In the random group, the CS was presented randomly with the US, whereby the onset of the CS varied between 72 and $288 \mathrm{~s}$ after onset of the US. The interval between the US onsets was $454 \mathrm{~s}$. The total number of trials per group was 10 . SEPs were recorded for every US presentation during the fear-conditioning session.

In order to familiarize the animals with the sugar pellets (Dustless Precision Pellets Sucrose $45 \mathrm{mg}$, Bio-Serv, Frenchtown, NJ, USA) used in Phase 2 of the experiment, four sugar pellets were fed directly after the fear-conditioning session, at the end of the fear-conditioning day and approximately $2 \mathrm{~h}$ prior to the first session of Phase 2 .

\subsubsection{Phase 2: apparatus}

The Phase 2 sessions were performed in the same experimental room as the Phase 1 sessions under similar conditions. The sessions were performed in a Plexiglas box $(41 \times 31 \times 20 \mathrm{~cm})$ different from the fear-conditioning box. A plastic tube provided access from outside the box to a food magazine mounted in a corner of the box. Two speakers in the covering lid of the box presented the CS. Similar to the Phase 1 conditions, polystyrene plates shielded the box, only leaving access to a camera, recording the animal's behavior.

\subsubsection{Phase 2: procedure}

All Phase 2 sessions were performed during the "lights off' period. In order to make the animals more receptive to the sugar pellets presented during this phase, they were deprived of food $2 \mathrm{~h}$ before each session. Acclimatization before the actual start of the sessions was the same as in Phase 1 (see Section 2.2.2). For each animal, the start of the Phase 2 sessions was counterbalanced over the 4 days.

During the Phase 2 sessions, the same CS as used in the Phase 1 session was presented paired with the presentation of a sugar pellet. The sugar pellet was delivered to the foodmagazine through the plastic tube, $30 \mathrm{~s}$ after onset of the CS. The interval between the CS-sugar pellet presentations ranged between 180 to $480 \mathrm{~s}$. Uneaten pellets were taken away approximately $1.5 \mathrm{~min}$ before the next CS onset. 


\subsection{Recordings of the somatosensory-evoked potentials}

During Phase 1 of the experiment, SEPs were recorded in the freely moving animal over one channel, as described by Stienen et al. [14]. In brief, SEPs were recorded from the vertex electrode, the ipsi- and contra-lateral frontal sinus electrodes served as reference and ground, respectively. Signals were amplified 2000 times, band-pass filtered between 15 and $300 \mathrm{~Hz}$ and digitized online at $2000 \mathrm{~Hz}$. For each SEP trial, 72 subsequent data segments of 256 data points (25 ms pre-stimulus, $102.5 \mathrm{~ms}$ post stimulus) were recorded and averaged. During Phase 2, SEPs were not recorded since only $\mathrm{CSs}$ and no USs (i.e. SEP evoking stimuli) were presented.

\subsection{Data and statistical analysis}

Calculations were performed with the aid of Microsoft Excel 2000. Statistical analysis was performed with SPSS 11.0 for Windows. Differences were considered to be significant when $P<0.05$.

Freezing and reward-directed behavior were evaluated after the Phase 2 sessions using the camera recordings. Freezing behavior was defined as the absence of all visible movements with the exception of breathing movements and pendulum motion of the head, while the animal sat in a tensed posture. Pendulum motion has been described to appear under circumstances of emotional excitement, most often fear [7]. Reward-directed behavior was studied by determining the latency-time to, and the number of, magazine-visits in the first $120 \mathrm{~s}$ after onset of the CS. A magazine-visit was defined as every entrance of the animal's snout into the food-magazine. The latency-time to a magazine-visit was defined as the time between the onset of the CS and the first magazine-visit that followed within 120 s. When the first magazine-visit after onset of the CS occurred later than $120 \mathrm{~s}$, the latency-time was scored as 120 s. The $120 \mathrm{~s}$ limit was arbitrarily chosen. The behavioral data were scored blind with respect to the fear-conditioning paradigm used. The scorings of the experimenter [HvO] demonstrated a high correlation (Pearson's correlation coefficient $0.86, n=30, P \leq 0.01$ ) with the scorings of a second observer who was unaware of the aims and procedures of this experiment.

SEP data analysis was performed by visual determination of the early peaks of the Vx-SEP waveform. Peak amplitudes and latencies were stored on disk. Parameters extracted for analysis were the amplitude of the P15 with respect to its following negative deflection and its latency. Furthermore, the amplitude of the positive peak occurring at approximately $37 \mathrm{~ms}$ (P37) and its latency were included in the analysis because of its consistency within, as well as between, subjects. For both peaks, signal-to-noise ratios were calculated. The signal-to-noise ratio was defined as the amplitude value divided by the root mean square calculated for the pre-stimulus period as described by Stienen et al.
[14]. Peak amplitudes with a signal-to-noise ratio $<2$ were considered to be zero and therefore replaced by the root mean square of the pre-stimulus period. For two animals (one in each group), the SEP waveforms were inconsistent with respect to the other animals due to technical or experimental problems. These two animals were excluded from data analysis, with respect to the SEP data.

All data were statistically analyzed by a two-way repeated measurements analysis of variance (RMANOVA), with the repeated factor "trial-block" and fixed factor "group" followed by post hoc analysis whenever appropriate.

\section{Results}

\subsection{Behavioral data}

\subsubsection{Freezing behavior}

Neither the paired group nor the random-control group showed freezing behavior during the acclimatization period in the box prior to the start of Phase 2. After presentation of the CS, although both groups showed freezing behavior, there was a significant difference between the groups over the trial-blocks (Fig. 1A; group $\times$ trial: $F_{7,126}=5.636, P<$ 0.001). Both groups showed a significant decrease of freezing behavior over the trial-blocks (Fig. 1A; post hoc one-way RM-ANOVA, factor trial-block: paired group: $F_{7,63}=14.127, P<0.001$; random-control group: $F_{7,63}=$ 8.732, $P<0.001)$. The paired group showed significant more freezing behavior on trial-blocks $1-5$ and 6-10 when compared to the random-control group (Fig. 1A; post hoc independent samples $t$ test: $t_{18}=4.144, P=0.001$ and $t_{18}=$ $2.819, P=0.018$ respectively).

\subsubsection{Reward-directed behavior}

During Phase 2, both groups shifted the initial aversive response to the $\mathrm{CS}$ towards an appetitive response. Both groups showed a significant decrease in latencies to, and an increase in the number of, magazine-visits over time (Figs. $1 \mathrm{~B}$ and $\mathrm{C}$; factor trial-block: latency to magazine- visit: $F_{7,126}=25.607, P<0.001$; number of magazine-visits: $\left.F_{7,126}=19.507, P<0.001\right)$, but there was no difference in the acquisition rate of the appetitive response (group $\times$ trial: latency to magazine-visit: $F_{7,126}=1.609, P=0.139$; number of magazine-visits: $F_{7,126}=1.450, P=0.191$ ). However, the paired group showed significantly longer latencies to and significantly less numbers of magazinevisits compared to the random-control group (Figs. 1B and C; factor group: $F_{1,18}=6.873, P=0.017$ and $F_{1,18}=$ 28.372, $P=0.048$ respectively).

\subsection{Somatosensory-evoked potentials}

The grand average waveform of the Vx-SEP with its peak definitions is shown in Fig. 2A. The two groups 
$\longrightarrow$ paired group
$\longrightarrow$ random-control group

A
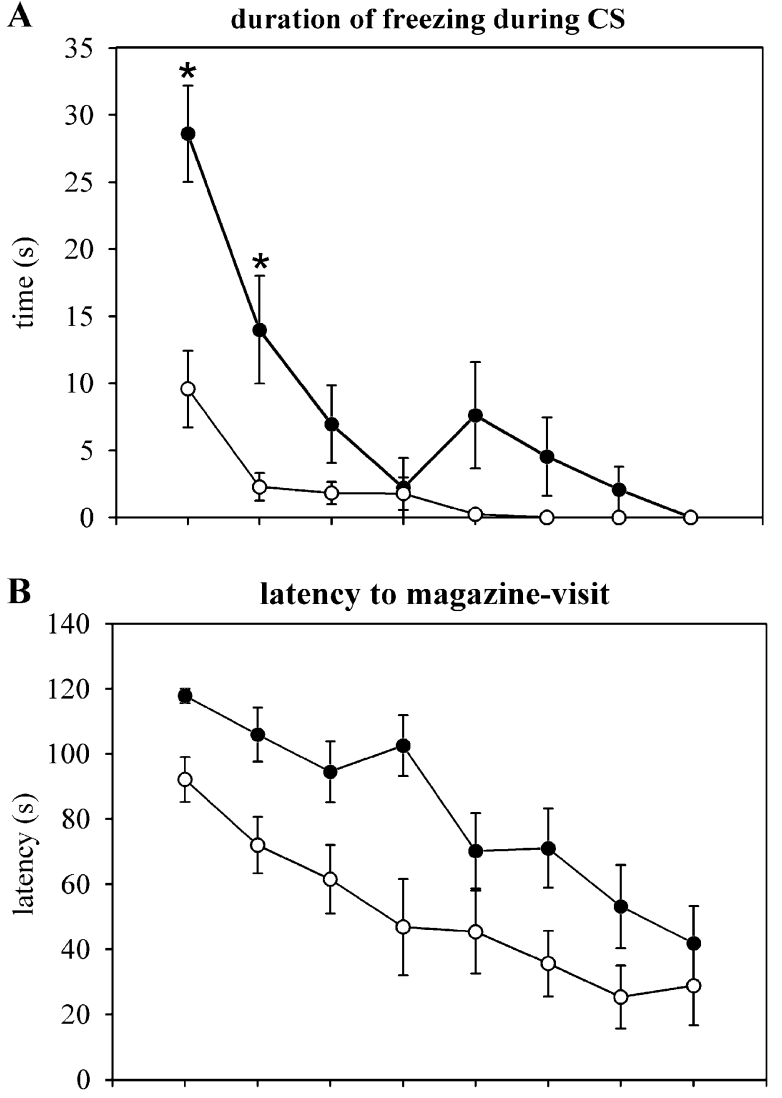

C

C number of magazine visits during CS

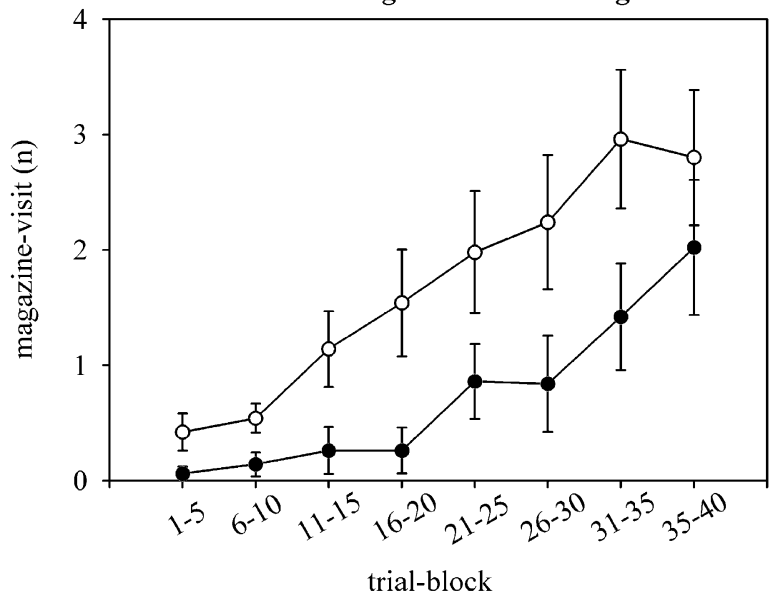

Fig. 1. Aversive and appetitive behavioral responses during Phase 2. (A) Duration of freezing behavior in the paired and random-control group during the CS presentation. Data are presented as the mean duration of freezing behavior (s) \pm SEM $(n=10)$ over the subsequent trial-blocks. Trial-blocks with a significant difference between the paired and randomcontrol group are indicated by *; (B) latency-time to the first magazinevisit in the paired and random-control group. Data are presented as the mean latency the first magazine-visit (s) $\pm \operatorname{SEM}(n=10)$ over the trialblocks; (C) number of magazine-visits during the CS presentation. Data are presented as the mean magazine-visits $\pm \operatorname{SEM}(n=10)$ over the trial-blocks.
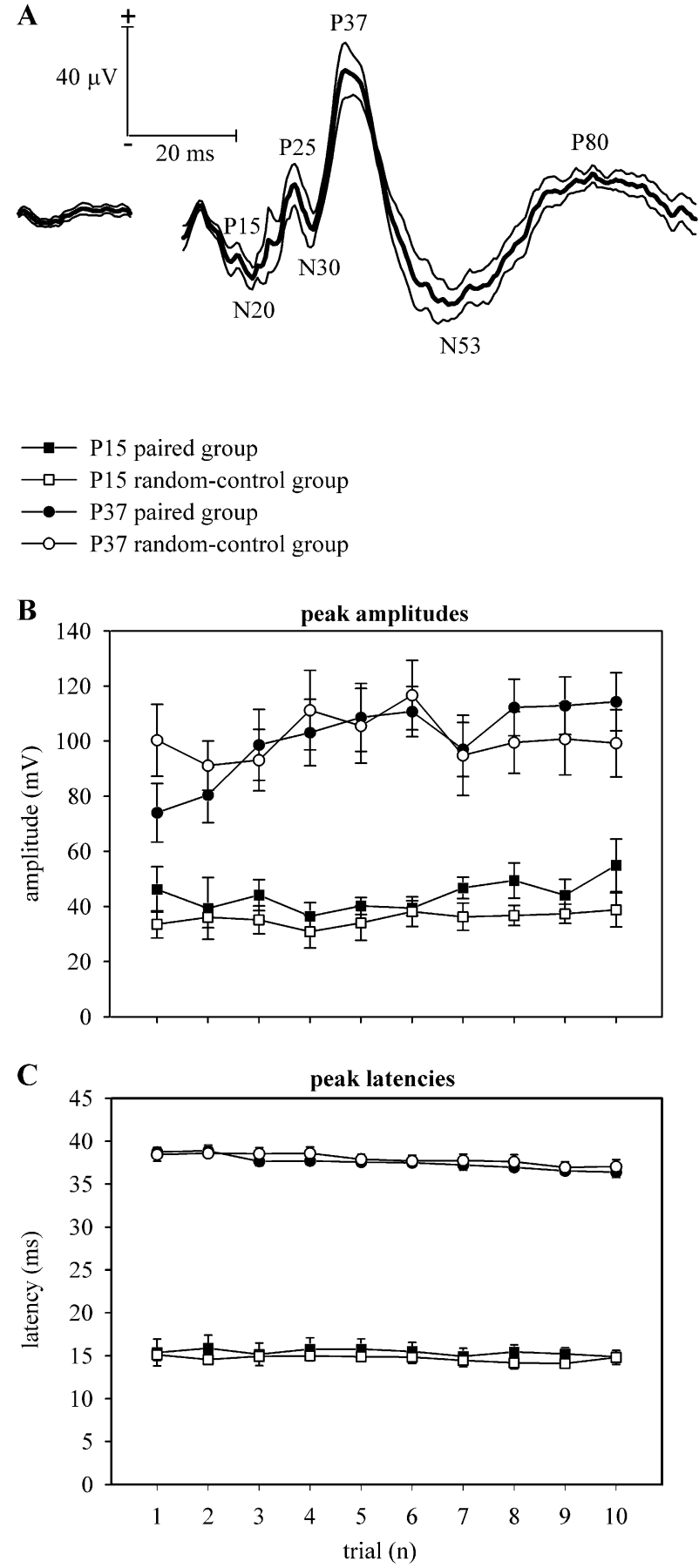

Fig. 2. Effect of fear-conditioning on the Vx-SEP during Phase 1. (A) Grand average waveform of the Vx-SEP-signals obtained from the random-control group during the first fear-conditioning trial. Data are presented as the mean (bold line) $\pm \operatorname{SEM}(n=9)$ (plain line). The curve interruption denotes the stimulus onset. Positive deflections (P) and negative deflections $(\mathrm{N})$ are accompanied by their average latency of onset (ms); (B) effect of fear-conditioning on the amplitudes of the P15 and P37 of the Vx-SEP. Data are presented as mean $(\mu \mathrm{V}) \pm \operatorname{SEM}(n=9)$ per trial; (C) effect of fear-conditioning paradigms on the latencies of the P15 and P37 of the Vx-SEP. Data are presented as the means $(\mathrm{ms}) \pm \operatorname{SEM}(n=9)$ per trial. 
showed no significant differences in the P15 amplitude and latency over the trial-blocks (Figs. 2B and C; trial $\times$ group, factor trial-block, and factor group: amplitude P15: $F_{9,144}=$ $0.551, P=0.835 ; F_{9,144}=1.290, P=0.247$; and $F_{1,16}=$ $2.032, P=0.173$, respectively; latency P15: $F_{9,144}=0.903$, $P=0.525 ; F_{9,144}=1.313, P=0.235$; and $F_{1,16}=1.518, P=$ 0.236 , respectively).

There were no differences between the two groups in P37 amplitude and latency over the trial-blocks (Figs. 2B and $\mathrm{C}$; trial $\times$ group and factor group: amplitude P37: $F_{9,144}=1.723, P=0.089$ and $F_{1,16}=0.000, P=0.998$, respectively; latency P37: $F_{9,144}=0.739, P=0.673$ and $F_{1,16}=7.376, P=0.586$, respectively). However, for both groups, a significant increase in amplitude and a significant decrease in latency were found over trials (Figs. 2B and C; factor trial-block: $F_{9,144}=3.711, P=0.009$ and $F_{9,144}=$ 7.928, $P<0.001$, respectively).

\section{Discussion}

In the present study, we investigated whether the SEPstimulation paradigm provides an effective US in Pavlov fear-conditioning and whether the Vx-SEP P15 and P37 were affected by the fear-conditioning paradigm. The principal findings are, first, that the paired group showed significantly more freezing behavior and significantly less reward-directed behavior compared to the random group. Second, we found that fear-conditioning itself did not affect the Vx-SEP P15 and P37. The behavioral findings strongly suggest that the paired group made a stronger association between the CS and the US during Phase 1 when compared to the random-control group and thus is supportive of the fact that the SEP-stimulation paradigm activates peripheral fibers involved in nociceptive processing. The neurophysiologic findings imply that, in future studies, using the present model for investigating drug-induced changes in SEPs and fear-conditioned behavior, the changes in the Vx-SEP components can be interpreted as drug-induced changes and not fear-conditioning-induced changes. Based on these findings, we conclude that the rat model, as developed in this study, can be used to study changes in the Vx-SEP components in relation to animal pain. In the future, such studies can be performed by fear-conditioning animals under different levels of analgesia, using the present model, which is potentially useful to validate the Vx-SEP components as potential indicator of the quality of analgesia.

Peck and Bouton [9] found an important effect of fearconditioning to context, during Phase 1 of an aversive-toappetitive transfer, upon the behavioral response shown during Phase 2. We suggest that fear conditioning to context did not affect the behavioral responses found in this study. First, although the context of the experimental room did not differ between the two phases in this experiment, the box used in Phase 1 differed, with respect to context, to the box used in Phase 2. Second, in the acclimatization period prior to the start of Phase 2, freezing behavior was absent in both groups and only occurred after the CS presentation.

The random-control group showed freezing behavior after presentation of the CS, during the first trial-blocks, although to a much lesser extent than the paired group. A likely explanation for the freezing behavior in the randomcontrol group is that in this group some of the CSs overlapped the US presentation due to randomization of the CS presentation. During these trials, the animals possibly made an association between the CS and the US. Animals in a random-control group making a CS-US association, although not as strong as animals in a paired group, have been described $[6,8]$.

The paired group showed less reward-directed behavior, compared to the random-control group. This finding is consistent with other studies and is explained in terms of excitatory aversive and appetitive motivational brain systems inhibiting one another $[9,10]$. According to this explanation, the CS presented during Phase 2 of an aversive-to-appetitive transfer paradigm activates the motivational aversive brain system, which has an inhibitory effect upon the motivational appetitive system, thus blocking learning an appetitive-conditioning task. Therefore, the appetitive system in the paired group, in the present study, may have been blocked to a greater extent resulting in less reward-directed behavior. Alternatively, differences in reward-directed behavior found in this study could also be a result of response competition at the motor level $[9,10]$. In this view, reward-directed behavior can be seen as an indirect measure of freezing behavior, indicating a stronger association between the CS and the SEP-stimulation paradigm in the paired group when compared to the random-control group.

Since every CS during Phase 2 was reinforced by the presentation of a sugar pellet, the possibility exists that the animals showed an appetitive response towards presentation of the sugar pellet itself rather than to the CS. The graph shown in Fig. 1B could possibly be interpreted this way, since in both groups all the latencies to magazine-visits were approximately $30 \mathrm{~s}$; the time at which the sugar pellet was dropped. However, two animals in the random-control group did not show an appetitive response in any of the 40 trials of the aversive-to-appetitive transfer paradigm, although the amount of freezing behavior was comparable to the animals that did show an appetitive response. When excluding the data of the two animals, the mean latencytimes (s) \pm SEM $(n=8)$ were: trial-block 26-30: $23.95 \pm$ 8.08, trial-block 31-35: $11.35 \pm 2.73$, trial-block 36-40: $11.95 \pm 3.49$ ). This indicates that the animals responded (at least in the later trials) to the CS rather than to the presentation of the sugar pellet itself.

The Vx-SEP components in the latency range studied are considered to be nociceptive in nature $[14,15]$, which is of primary importance when using these components as indicators of analgesic efficacy. Nociceptive processing involves primarily activation of A $\delta$-fibers (acute pain) and 
C-fibers (slow pain). It is known that electrical stimulation as used in the present study activates $A \alpha$-fibers involved in motor processing, $\mathrm{A} \beta$-fibers involved in mechanosensation, and $\mathrm{A} \delta$ - and $\mathrm{C}$-fibers $[3,4,11,12]$. Based on the conduction velocities $(A \alpha:$ 60-70 m/s, A $\beta: 30-40 \mathrm{~m} / \mathrm{s}, A \delta: 4-30 \mathrm{~m} / \mathrm{s}$, and $\mathrm{C}: 0.4-1.8 \mathrm{~m} / \mathrm{s})[3,4,11,12]$ and the distance between the stimulus location and the recording site in this study (approximately $0.15-0.20 \mathrm{~m}$ ), both $\mathrm{A} \beta-$ and $\mathrm{A} \delta$-mediated responses can be found within the latency range examined in the present study. It has been shown that the Vx-SEP P15 [15] and P37 (unpublished observations, PJ Stienen) are affected by the $\mu$-opioid receptor agonist fentanyl. Since $\mu$ opioid receptor agonists selectively modulate nociceptive processing (A $\delta$ - and $\mathrm{C}$-fibers) on both peripheral and supraspinal level but not tactile processing (A $\beta$ fibers) $[5,13]$, we consider both the Vx-SEP P15 and P37 to be A $\delta$-mediated. The fact that the animals in this study clearly demonstrated fear-induced behavior after presentation of the CS further supports this hypothesis.

In conclusion, the findings obtained in the present study strongly suggest that the SEP-stimulation paradigm used forms an effective US in a Pavlovian fear-conditioning paradigm and that the Vx-SEP P15 and P37 are not affected by fear conditioning itself. This provides us with a unique tool to study the direct relationship between the characteristics of the Vx-SEP components and animal pain. Therefore, the rat model developed in this study is potentially useful for validation of the Vx-SEP P15 as parameter of the level of analgesia.

\section{References}

[1] Z. Annau, L.J. Kamin, The conditioned emotional response as a function of intensity of the US, J. Comp. Physiol. Psychol. 54 (1961) $428-432$.
[2] M.E. Bouton, Context, time and memory retrieval in the interference paradigms of Pavlovian learning, Psychol. Bull. 114 (1993) 80-99.

[3] B. Bromm, J. Lorenz, Neurophysiological evaluation of pain, Electroencephalogr. Clin. Neurophysiol. 107 (1998) 227-253.

[4] M.G. Fehlings, C.H. Tator, R.D. Linden, I.R. Piper, Motor and somatosensory-evoked potentials recorded from the rat, Electroencephalogr. Clin. Neurophysiol. 69 (1988) 65-78.

[5] J. Kalliomäki, X.-L. Luo, Y.-B. Yu, J. Schouenborg, Intrathecally applied morphine inhibits nociceptive $\mathrm{C}$ fiber input to the primary somatosensory cortex (SI) of the rat, Pain 77 (1998) 323-329.

[6] R.J. Keller, J.J.B. Ayres, W.J. Mahoney, Brief versus extended exposure to truly random control procedures, J. Exp. Psychol., Anim. Behav. Processes 3 (1977) 53-65.

[7] V.G. Kolpakov, P.M. Borodin, N.N. Barykina, Catatonic behaviour in the Norway rat, Behaviour 62 (1977) 190-208.

[8] E.F. Kremer, Truly random and traditional control procedures in CER conditioning in the rat, J. Comp. Physiol. Psychol. 76 (1971) $441-448$.

[9] C.A. Peck, M.E. Bouton, Context and performance in Aversive-toAppetitive and Appetitive-to-Aversive transfer, Learn. Motiv. 21 (1990) 1-31.

[10] M.J. Scavio, Classical-classical transfer: effects of prior aversive conditioning in rabbits (Oryctolagus cuniculus), J. Comp. Physiol. Psychol. 86 (1974) 107-115.

[11] J. Schouenborg, J. Kalliomäki, P. Gustavsson, I. Rosén, Field potentials evoked in rat primary somatosensory cortex (SI) by impulses in cutaneous A $\beta$ - and C-fibres, Brain Res. 397 (1986) $86-92$.

[12] F.Z. Shaw, R.F. Chen, C.F. Yen, Dynamic changes of touch- and laser heat-evoked field potentials of primary somatosensory cortex in awake and pentobarbital-anesthetized rats, Brain Res. 911 (2001) $105-115$.

[13] S.C. Silbert, D.W. Beacham, E.W. McCleskey, Quantitative single-cell differences in $\mu$-opioid receptor mRNA distinguish myelinated and unmyelinated nociceptors, J. Neurosci. 23 (2003) 34-42.

[14] P.J. Stienen, Z.L. Haberham, W.E. van den Brom, H.N.M. de Groot, A.J. Venker-van Haagen, L.J. Hellebrekers, Evaluation of methods for eliciting somatosensory-evoked potentials in the awake, freely moving rat, J. Neurosci. Methods 126 (2003) 79-90.

[15] P.J. Stienen, W.E. van den Brom, H.N.M. de Groot, A.J. Venker-van Haagen, L.J. Hellebrekers, Differences between primary somatosensory cortex- and vertex-derived somatosensory-evoked potentials in the rat, Brain Res. 1030 (2004) 256-266. 